\title{
RELACION DE LA CADENA DE VALOR DEL SECTOR AGRÍCOLA, SUS BENEFICIOS FINANCIEROS- MEDIOAMBIENTALES Y ELTURISMO SOSTENIBLE
}

\section{RELATION OF THE VALUE CHAIN OF THE AGRICULTURAL SECTOR, ITS FINANCIAL-ENVIRONMENTAL BENEFITS AND SUSTAINABLE TOURISM}

\author{
Mag. Alain Castro-Alfaro*, PhD. Helber Ferney Guzmán-Ramos** \\ Mag. Jairo Jamith Palacios-Rozo ** , Ing. Iván Fernando Amaya-Cocunubo ** \\ Mag. Francisco Javier Lagos-Bayona ${ }^{* *}$ \\ * Corporación Universitaria Rafael Núñez, Facultad de Ciencias Contables y \\ Administrativas, Programa de Contaduría Pública, Grupo de Investigación Cartaciencia \\ Cartagena, Bolívar, Colombia \\ $+57-3006149476$ \\ E-mail: alain.castro@curnvirtual.edu.co \\ ** Universidad Colegio Mayor de Cundinamarca \\ Bogotá, Cundinamarca, Colombia, Grupo de Investigación CYGA \\ E-mail: (hfguzmanr, jjpalacios, ifamaya, lagos)@unicolmayor.edu.co
}

Resumen: Establecer la relación del efecto en una cadena de valor agrícola local, gracias a los beneficios obtenidos de tipología social, cultural, medioambiental y económica, a partir de diferentes actividades en el marco del turismo, permite determinar la contribución al producto interno bruto PIB, entendido como un indicador económico que mide la producción en el interior de un país, así como su impacto, obtenido entre los años 2010 y 2018. A partir de estos resultados, será posible establecer un método de análisis que permita en futuros estudios e investigaciones, verificar el impacto del turismo en diversas cadenas de valor.

Palabras clave: Sostenibilidad, cadena de valor, producto interno bruto, turismo sostenible, beneficios financieros

\begin{abstract}
Establishing the relation of the effect in a local agricultural value chain, thanks to the benefits obtained from social, cultural, environmental and economic typology, from different activities in the framework of tourism, allows determining the contribution to the GDP, understood as an economic indicator that measures production in the interior of a country, as well as its impact, obtained between 2010 and 2018. From these results, it will be possible to establish an analysis method that allows future studies and research to verify the impact of tourism on various value chains.
\end{abstract}

Keywords: Sustainability, value chain, gross domestic product, sustainable tourism, financial benefits

\section{INTRODUCCIÓN}

La ejecución de políticas, planes y programas creados para promocionar las actividades turísticas de la ciudad de Bogotá-Colombia y cuyo objetivo es posicionarla como un destino turístico sostenible y competitivo a nivel local, nacional e internacional, se convierte en una estrategia que permite el impulso del desarrollo económico (Instituto Distrital de Turismo-IDT, 2019). De igual manera, para este gobierno, el turismo se ha convertido en uno de los principales elementos en 
las políticas de economía naranja; así como el hecho de la recuperación de recursos turísticos que habían sido olvidados por encontrarse en zonas de conflicto armado, ha permitido no sólo el acceso a las mismas, si no toda una campaña de promoción que implica la seguridad para los turistas ya sean nacionales o extranjeros.

A su vez, es menester acrecentar el tema de la Responsabilidad social ya que esta se fundamenta en la competitividad y para ello es necesario llevar a cabo estrategias en pos de ella. Ahora, en Colombia a partir de la década pasada se ha convertido en uno de los países donde se genera inversión internacional, donde los inversionistas lo consideran como como uno de los destinos preferidos (Blanco, 2016). Ante lo dicho, el pensamiento estratégico, el cual según Bernal (2018), es un arte para la formulación, implantación y evaluación de decisiones para que la empresa pueda conseguir sus objetivos, debe permear en las organizaciones con el fin de prever y establecer los dinamismos que las organizaciones deben tomar para logar el éxito en el mercado (García, Paz y Cantillo, 2019; Castro, 2018). A su vez, entendiendo que la competitividad es uno de los elementos que mayor incide en la motivación y que es de ayuda a que los trabajadores de un ente organizativo mantengan un alto nivel de productividad (Cardona, Lamadrid y Brito, 2018; Gómez, Bohórquez y Severiche, 2018). Finalmente, implica un elevado grado de responsabilidad con sus consumidores quien al final se debe la organización (Díaz y Bernal, 2017).

A través del Observatorio de Turismo, se tiene acceso a la identificación y caracterización de los productos turísticos, lo cual destaca el valor de las ofertas de la ciudad, así como el impacto económico en ésta, siendo así una fuente de información que permite al sector tomar decisiones de diferente tipo (Instituto Distrital de TurismoIDT, 2019); entre éstos hallamos como principales productos los de índole histórico, cultural, de arte, religioso, arquitectónico, turismo de naturaleza, gastronómico, urbano, de salud y bienestar, deportivo; asimismo, eventos nacionales e internacionales tales como festivales de teatro, encuentros de diversos géneros de música y los constituidos como festivales al parque, además, las actividades nocturnas programadas de tipo comercial o de esparcimiento como la ciclovía, entre otros, son actividades de costo viable para los turistas, lo que hace a Bogotá en uno de los mejores destinos para esta vigencia en particular.
Ahora bien, Serrano, Montoya \& Cazares (2018), destacan la relevancia de este tipo de enfoques como una herramienta de optimización en cuanto a la calidad de los servicios turísticos, así como el posicionamiento y crecimiento económico del lugar donde sea usado en cuanto a sostenibilidad turística se refiere. Es así como la competitividad, se convierte en un indicador de evaluación en relación a la rentabilidad del sector turístico en un país, no obstante, en algunos casos si bien las estrategias implementadas tienen éste fin, las entidades de tipo gubernamental los obvian sin causa aparente.

Por su parte, los autores Pardavila \& Navarro (2014), integran tres condiciones en el marco del desarrollo sostenible: los conceptos de calidad de vida, desarrollo y bienestar social; mayor igualdad en la distribución de la riqueza y, determinan que es casi que obligatorio, realizar un uso racional de los recursos naturales, ya que, de lo contrario, habitar en el planeta será imposible a largo plazo. Paz, Castro \& Palacios (2019), afirman que el emprendimiento es una actividad que se concibe como una oportunidad que aporta al desarrollo socio-económico de los países, teniendo presente que es un deber de los emprendedores el satisfacer las necesidades del cliente por medio de un valor definido, el cual es el servicio que la empresa ofrece (Caballero, 2016; Bernal, 2017).

Para el caso de Samaná en República Dominicana, se logró demostrar que, a pesar del incremento de la zona hotelera, no se evidencia que sea una actividad económica sostenible, debido a que no cumplen las normas ambientales establecidas, estudio con base en análisis de varios factores, entre ellos la competencia del Estado para la conservación del entorno, así como los diversos impactos que pueden ser sociales, económicos y ambientales y la nula acción que permita mitigar o compensar.

Del mismo modo, Molina \& Font (2016), señalan que se aumenta la fiabilidad de los resultados a través de los métodos mixtos, brindando una visión transformadora en términos de cambio social, evidenciando que son de gran valor y por ello, se requiere una mayor comprensión para su utilización por parte de académicos e investigadores en esta rama específica del turismo sostenible.

En tal sentido, se identifica la necesidad de correlacionar el efecto en una cadena de valor local de actividades turísticas que sean sostenibles, en 
este caso para el sector agrícola, determinando la contribución al producto interno bruto PIB y su impacto en la ciudad de Bogotá-Colombia, tomando datos del período comprendido entre los años 2010 a 2018. Los resultados ofrecerán un método de análisis para futuras investigaciones académicas, acerca del impacto del turismo en diferentes cadenas de valor, así como su posible réplica en otras ciudades con una conducta similar.

\section{METODO}

En la base de datos SCOPUS, se identificó en los meses completos de marzo y abril e inicios de mayo, un marco muestral de revistas académicas especializadas de acceso abierto, así: 63 del área General Economics, Econometrics and Finance; 22, del área temática Tourism, Leisure and Hospitality Management y 41 del área temática Renewable Energy, Sustainability and the Environment.

En ese orden de ideas, en ScienceDirect mediante cadenas de búsqueda con las siguientes palabras clave: "tourism and agricultura", "sustainable tourism", "agriculture and sustainable tourism", "sustainable touristic practices"; "turismo sostenible en Bogotá Colombia" y "agricultural value chain in Colombia, se ejecutó la búsqueda de libros, capítulos de libros, artículos de revisión y de investigación, en idiomas español, inglés y portugués. De mismo modo, se efectuaron lecturas de noticias y documentos resultados de investigaciones por parte de la Secretaría de Desarrollo Económico Observatorio de Turismo del IDT y la Alcaldía de Bogotá.

Tabla 1 Identificación de los artículos

\begin{tabular}{|c|c|c|c|}
\hline $\begin{array}{l}\text { Intervalo } \\
\text { temporal }\end{array}$ & $\begin{array}{l}\text { Campos } \\
\text { de } \\
\text { informaci } \\
\text { ón }\end{array}$ & Palabras clave & $\begin{array}{c}\text { No. } \\
\text { artículos } \\
\text { científicos }\end{array}$ \\
\hline \multirow{5}{*}{$\begin{array}{l}2010- \\
2020\end{array}$} & \multirow{5}{*}{$\begin{array}{l}\text { Título; } \\
\text { Resumen- } \\
\text { abstract; } \\
\text { Palabras } \\
\text { clave- } \\
\text { keywords }\end{array}$} & $\begin{array}{c}\text { sustainable } \\
\text { tourism }\end{array}$ & 52 \\
\hline & & $\begin{array}{c}\text { tourism and } \\
\text { agricultura }\end{array}$ & 148 \\
\hline & & $\begin{array}{c}\text { agriculture and } \\
\text { sustainable } \\
\text { tourism }\end{array}$ & 52 \\
\hline & & $\begin{array}{l}\text { agricultural } \\
\text { value chain in } \\
\text { Colombia }\end{array}$ & 660 \\
\hline & & $\begin{array}{l}\text { sustainable } \\
\text { touristic } \\
\text { practices } \\
\end{array}$ & 208 \\
\hline
\end{tabular}

\begin{tabular}{|c|c|c|c|}
\hline & turismo & 166 \\
& & sostenible en & \\
& Bogotá & \\
& Colombia & \\
\hline
\end{tabular}

Fuente: Elaboración propia

Se procede a la lectura inicial del título, resumen y palabras clave, a continuación a la elección de los artículos que cumplen con el criterio 1, con el criterio 2 o con ambos criterios, así: 1) Relacionar en el título alguna de las palabras clave y 2) Relacionar en el abstract y/o en el contenido las palabras clave o relacionarse directamente con el tema, para un resultado de 119 artículos de un total de 1286 artículos, lo que equivale a un $9.25 \%$, se identifican los que poseen mayor número de citas, los que sus autores cuentan con un mayor número de publicaciones en el marco muestral, los que contienen información directamente relacionada con el tema y los más recientes; ya posterior a la lectura de los resúmenes y en algunos casos de la introducción, son elegidos para la revisión 21 artículos, excluyendo los que no incluían el objetivo de la investigación.

Finalmente, se procede a la lectura completa de los 21 artículos y posteriormente al análisis del contenido con base en la siguiente información:

a) Objetivo(s);

b) Problemática;

c) Contexto;

d) Metodología;

e) Resultados destacados;

f) Conclusiones;

g) Recomendaciones y sugerencias.

\section{RESULTADOS}

\section{Turismo y agricultura}

Mediante la metodología Delphi, Bokelmann \& Ramírez (2017), eligen los indicadores que permitirán el análisis del desarrollo rural para los siete legados y patrimonios del campesinado colombiano: cultural, social, físico, humano, natural, económico e institucional. Este documento pretende relacionar el marco y su utilidad por primera vez en el ámbito del desarrollo rural, no obstante, es de importancia la identificación en los indicadores de los puntos fuertes y los que no lo son, así como la relación con los legados para consolidar el estudio, asimismo, la aplicación de otros estudios en otros niveles, ya que sólo se tomó la percepción de campesinado seleccionado como fuente de información.

En cuanto a la situación de inseguridad alimentaria que se presenta a nivel mundial Ambelu, Lovelock 
\& Tucker (2018), plantean como propuesta, que el turismo se constituya en la base para la generación de nuevos estilos de vida, lo que logra mediante un modelo que lo integra y establece su relación a partir de estudios previos, con la pobreza, sostenibilidad, subsistencia, y seguridad alimentaria, llegando a la conclusión de que mediante diferentes mecanismos a través del turismo, se puede disminuir significativamente la pobreza, cuya erradicación en todas sus formas y dimensiones se espera para el año 2030 en el marco de los Objetivos de Desarrollo Sostenible ODS, igual que la seguridad alimentaria, ya que para el mismo año no debe existir hambre ni desnutrición, tarea enfocada en la promoción de prácticas agrícolas sostenibles; dichos autores también resaltan como se ha visto en muchas otras revisiones, la importancia de la participación a nivel gubernamental, niveles sectoriales, políticas y normas que de alguna manera conlleven no sólo el cumplimiento de las mismas, sino la óptima interacción de los diferentes agentes.

Addinsall, Weiler, Scherrer \& Glencross (2017), determinan al turismo agroecológico como una vía para mejorar los medios de subsistencia de los pequeños agricultores en Pentecostés del Sur, Vanuatu, esto gracias a los objetivos de conservación y seguridad alimentaria, que si bien la agricultura tradicional lo ha cubierto en algunos aspectos, así como en zonas específicas, no debe desconocerse que debe propenderse a la interacción a nivel económico, ambiental y sociocultural. A nivel rural, se ha impulsado actividades turísticas tales como el ecoturismo y el agroturismo, lo que favorece no sólo el desarrollo sino la conservación de dichas áreas, sin embargo, se identificó la deforestación en una zona específica, debido al uso de corte y quema como método de jardinería. Esto quiere decir, que aunque se reconoce el valor agregado que puede tener el turismo agroecológico para los agricultores, no hay suficientes estudios, proyectos o investigaciones a nivel descriptivo y práctico que abarque su utilidad a nivel político, económico y sociocultural, por lo cual los autores recomiendan seguir esta línea de investigación y se permita así una mayor apropiación tanto del concepto como la aplicación del mismo.

Un análisis relacionado con la agricultura urbana sostenible que precisamente se origina debido al crecimiento de las ciudades en los países en desarrollo y en la cual se incluye la acuicultura, la ganadería y las plantas, así como se integran actividades como limpieza y producción de alimentos frescos y nutritivos, sin embargo, tiene como desafíos el acceso a insumos de tipo agrícola, fertilizantes y agua, De Bon, Parrot \&Moustier (2010). Ahora bien, a pesar de que se puede reutilizar los residuos urbanos, dicha acción no tiene utilidades representativas, en cambio si se considera que presenta un riesgo de obtener productos que no sean seguros, desconocidos y además con peligrosidad desconocida; adicionalmente la agricultura urbana sostenible aún no es lo suficientemente promovida por los entes gubernamentales, a pesar de que si existen casos específicos, no son suficientes para concluir que logra el propósito para el que se originó. Es por esto, que a manera de recomendación se debería documentar todo estudio, revisión o investigación relacionada a la integración de la agricultura urbana en el desarrollo urbano, convirtiéndose en un elemento valioso para considerar en la sostenibilidad social, económica y ambiental.

Por último, Veldkamp, Altvorst, Eweg, Jacobsen, Kleef, Latesteijn, et al. (2009), identifican las transiciones del desarrollo sostenible del sector agrícola holandés, a través del programa de innovación Transforum, éste, opera en un marco analítico constituido por cinco hipótesis, determinando que el desarrollo sostenible es: una propiedad dinámica; necesita innovación; dicha innovación, consiste en un proceso de aprendizaje no lineal; demanda la participación de organismos del gobierno, organizaciones de la sociedad civil y comunidad empresarial, así como institutos o entes de conocimiento; ya por último el programa necesita que todos los actores o agentes participen de manera transdisciplinar, queriendo así convertirse en una organización de aprendizaje, aunque existan las diversas normas que no permiten el desarrollo del sector agrícola rural en dichos países. Este marco conformado por las hipótesis mencionadas, es el que va a permitir medir y evidenciar que el sector agrícola debe encaminarse hacia un desarrollo sostenible, con todo lo que esto conlleva.

Es así que su objetivo es llevar a cabo esa transición de la agricultura de los Países Bajos por medio del desarrollo sostenible, superando las barreras institucionales y burocráticas que encuentre y así pueda lograr consolidarse no sólo como un programa exitoso, sino en uno de replicación para las diversas comunidades a nivel mundial y de gran espectro.

Es posible concluir que la mayoría de estudios se enfocan en el desarrollo sostenible como clave para el crecimiento económico y desarrollo de una 
comunidad o nación. Si bien se observan casos exitosos, se evidencian situaciones que siguen presentándose en el marco de los procesos que involucran actividades humanas en interacción con lo que lo rodea.

\section{Turismo sostenible}

El turismo sostenible debe razonarse como un elemento en el desarrollo de la responsabilidad social empresarial, Lalangui, Espinoza \& Pérez (2017), encargado de hallar el equilibrio entre las actividades desarrolladas en el marco de las actividades turísticas con el medio ambiente y sus recursos. Es así, como se establecen tres características para que sea considerado sostenible: ambientalmente respetuoso (uso racional de recursos, preservación y respeto a espacios protegidos), socialmente justo (respeto a la cultura de la comunidad y preservación de la autenticidad) y económicamente viable (repartición equitativa de ganancias, reducción de la pobreza, mejoramiento de calidad de vida y beneficios de tipo económico), un ejemplo para este último son las cifras reportadas en las estadísticas de la Organización Mundial del Turismo OMT correspondiente al año 2016, donde se visualiza la creación de 250 millones de puestos de trabajo y una facturación superior a los 510 millones de dólares, lo que equivale a un $10 \%$ del PIB mundial. Aunque se aprecian buenos resultados, no se puede determinar que los otros pilares alcanzaron efectos al mismo nivel o de forma similar.

Asimismo, Amaya, Guzmán y Salazar (2018), determinan que tanto la responsabilidad social empresarial como el turismo sostenible están relacionados, favoreciendo uno directamente al otro, adicional, por medio de indicadores cuantitativos y cualitativos, aportan tanto al campo académico como al práctico, en atención a que la documentación de los procesos, protocolos y/o proyectos a niveles públicos y privados para la actividad turística, se impulsam a otros agentes del campo a realizar actividades que contribuyan a lograr un turismo sostenible real.

Por otra parte, Salom (2020), expone un análisis sobre la aplicación del principio de turismo sostenible y la adopción formal mediante resolución firmada en octubre de 2019, por parte de la Convención Marco sobre Ética del Turismo, y en la que establece en su artículo 2, que a través de los principios éticos se promoverá un turismo responsable, de acceso libre y sostenible, que será vigilado por todos los que han sido denominados agentes de desarrollo, entre los que se encuentran los gobiernos nacionales, locales específicos, entidades financiadoras de proyectos, establecimientos, empresas y asociaciones turísticas, organizaciones sindicales, profesionales y empleados del sector, así como también turistas, excursionistas, población local y ONG especializadas en el campo.

Asimismo, concluye que, gracias a la adecuada planificación y gestión de las actividades turísticas, se puede obtener tanto el crecimiento económico como la promoción del desarrollo sostenible, así se determina que es necesario plantear enfoques a nivel regional en atención a las particularidades de cada zona, ya que si bien las políticas y leyes son una guía que dirige hacia dicho desarrollo, no deben olvidarse las estrategias y herramientas que aportan en la creación de buenas prácticas.

Lagos, Sánchez \& Palacios (2019), destacan como una ciudad como Mompox que, gracias a sus tradiciones, arquitectura, entre otros, ha logrado convertirse en un destino turístico sostenible con certificado de calidad del ICONTEC, todo gracias a modelos de gestión social, que podrían ser aplicados en otras ciudades y así lograr constituir todas estas ciudades consideradas destinos turísticos, en fuentes de sostenibilidad para la ciudad y por ende para el país.

Ahora bien, Pulido, J. \& Pulido M. (2015), demuestran la necesidad de crear incentivos que permitan el desarrollo de modelos fundamentados en producción y consumo turístico sostenible por parte de los gobernantes, sin dejar de lado, que el uso de materia y energía debe ser menor, en atención a que no se observan adelantos en materia de sostenibilidad del turismo, lo que evidencia una vez más, que a pesar del cambio en el enfoque, ha sido insuficiente para obtener mejores y más resultados, observando que prácticamente se continúa en el mismo modelo.

Sin embargo, identifican en la literatura, ocho argumentos que permiten considerar la sostenibilidad como pieza clave en el desarrollo de la actividad turística, estableciendo la sostenibilidad para la consolidación del turismo como estrategia de desarrollo, la competitividad, como medio para dar respuesta a la masificación y a la gestión adaptativa de los destinos así como reformulación de su imagen, reducción de costos en la industria de este campo, para la consecución de un nuevo turista y para garantizar una cultura de convivencia; reiterando la importancia participativa de los agentes involucrados y la administración. 
Alcaraz et al (2017), a partir de entrevistas con veintiocho organizaciones del sector de hostelería en tres países iberoamericanos, exploraron generalidades en torno a responsabilidad social y sostenibilidad ambiental por parte de los gestores del área de recursos humanos, allí identificaron empresas que, desde su cultura institucional, les interesa y se encuentran realmente comprometidas con la sostenibilidad. De igual manera, crearon un modelo que permite a los encargados de estas áreas involucrar en este contexto empresarial temas como la identificación de factores socio ecológicos que de alguna forma afecten el presente y futuro, la búsqueda de estrategias o herramientas que fomenten tanto a empleados como a externos involucrados su participación en actividades sostenibles, priorización de iniciativas y su implementación, entre otros aspectos relevantes para que realmente se forme esa cultura sostenible dentro de la organización.

Usando a China como ejemplo, Weaver, Tang \& Zha (2020), demuestran que de la manera en que se encuentra planteado el Informe autorizado al $19^{\circ}$ Congreso Nacional del Partido Comunista de China, se requiere del poder y la política para obtener resultados asociados a la sostenibilidad, se establece que debe mejorarse la forma de vida de las personas, el alivio a la pobreza y la civilización ecológica, sin dejar de lado sus valores culturales. Este panorama no es diferente de lo que sucede en otras regiones del mundo; ejemplo de ello ocurre en el Parque Natural Chicaque, que, si bien tiene establecido acciones constantes que se han pasado por generaciones, requiere la intervención en los impactos ambientales, socioculturales y económicos, asimismo, en temas de delimitaciones de territorio, convirtiendo este modelo de negocio en una base para mejorar la calidad de vida y como fuente de crecimiento económico para el sector, como afirma González, Palacios \& Sandoval (2017) tomar decisiones e implementar cambios rápidamente marca la diferencia entre crecer o desaparecer; este atributo en una organización se denomina agilidad organizacional, ya que Niño \& Contreras (2020), establecen como acción de mejora a los planes ya implementados, la incorporación de los productos agrícolas en la cadena de abastecimiento, ya que la economía de estos sectores se basa en ella. Esta actividad se lleva a cabo a través de programas educativos ee integradores.

Se concluye entonces, que, desde el concepto adoptado de turismo sostenible a nivel personal, social y cultural, todas las acciones, estrategias, planes, programas y cualquier medio que acceda a que las actividades involucradas con el turismo, den paso a la verdadera concientización de integrarse a un sistema (diversos factores $y$ agentes), que permiten que la experiencia se convierta en una actividad de calidad humana, como entes encargados de la preservación de lo que nos rodea, así como a la dinámica del movimiento de las masas hacia un objetivo específico.

\section{Cadena de valor agrícola}

A partir del concepto básico de que el propósito de una cadena de valor es generar mayor beneficio a los consumidores y que se llegue a ser más competitivo, resultado de la satisfacción del usuario o cliente, por la adquisición de un producto o servicio, estas cadenas se convierten en una herramienta de planeación estratégica y análisis que puede ser aplicada en cualquier campo y a través de los resultados obtenidos, logre que los líderes o altas gerencias, tomen decisiones de diversos tipos: preventivo, correctivo o en algunos casos de mejora. Mediante la caracterización de una de estas cadenas de valor en el sector vitivinícola en pequeños y medianos productores de Baja California Andrade (2015), demostró, los problemas localizados a nivel de infraestructura productiva, así como uso de intermediarios en la comercialización y desabastecimiento, además de la ausencia de proveedores locales de insumos, de igual manera logró establecer la necesidad de inversión en cuanto a formación y capacitación del recurso humano, la escasez de agua e impuestos de costos altos; así como escaso financiamiento. Los análisis de estos descubrimientos hacen que la empresa pueda implementar acciones de tipo correctivo que le permitirán reducir costos en un momento dado, así como elevar su nivel de competitividad.

Así como toda cadena de valor genérica de una empresa, en sus actividades primarias se encuentran todas las actividades implicadas en la etapa de producción, mientras que las secundarias son de apoyo a las anteriores y constituyen los insumos tanto en infraestructura como en recurso humano, tecnología (uso o desarrollo) y ya funciones de tipo funcional. Es así que se constituyen las preguntas alrededor de la reducción de costos sin afectar la calidad del producto, la identificación de aquellas fases o etapas de la cadena es las que se presentan situaciones que se consideren problema, para ya proceder a instaurar las acciones correctivas respectivas. 
Asimismo, Guzmán, Amaya, Salazar y Ramírez (2018), resaltan que debido a la amplitud del sector turístico se incluye la importancia de la incidencia en la gestión productiva y comercial en sus relaciones con stakeholders, partiendo del principio de cadena de valor, establecido por Morrillo (2005), como el conjunto de actividades y comunidad generadoras de ganancias, que comprende desde la adquisición de fuentes de materias primas hasta que es entregado al consumidor final, el producto, bien o servicio.

Como iniciativa para el impulso del desarrollo agrícola, Scott (2014), en las actividades que se encuentran comprendidas entre el período de la cosecha y la entrega del producto, una matriz de factores que deben ser priorizados en las cadenas de valor, incorporando además la responsabilidad social empresarial RSE como elemento que permita mejorar la competitividad, teniendo en cuenta dos componentes: el producto y el lugar. Tales factores comprenden desde las ventas anuales, el comportamiento de los precios y costos, hasta las nuevas tecnologías desarrolladas, la mecanización de los procesos y la competitividad, entre otros; tales factores pueden elegirse de diferentes fases del proceso, así como manejar diversas variables, por lo cual, el éxito de la creación de la cadena de valor se encuentra en la elección de los factores o enfoques determinantes para hallar situaciones que no se deseen o que sean tendientes a mejora, logrando obtener el producto deseado de mejor calidad a bajo costo y minimizando el impacto al medio ambiente.

Ahora bien, los tratados de libre comercio han permeado la economía y se han centrado en esa búsqueda de estrategias y alianzas del sector productivo, público y privado.

En cuanto a la resiliencia operacional en cadenas de valor agrícolas tropicales, Aboah, Wilson, Rich, \& Lyne (2019), abarcan diferentes aspectos a nivel socioecológico, en cuanto las cadenas de suministro de materias primas y elementos básicos en la cadena de valor en sus fases iniciales. Para ello, es necesario que se analicen elementos tales como la colaboración y la adaptabilidad, adicionalmente la inventiva y la flexibilidad para el área económica, lo que se evalúa a través de indicadores de rendimiento. Sin embargo, se encuentra como limitación su aplicabilidad únicamente a cadenas de valor de tipo agrícola específicamente, que contemple actividades que no requieran el uso de tecnología. En la literatura siguen siendo escasos los estudios que involucren la resiliencia en cualquier tipo de cadena.

En relación a Suramérica, se encuentra un estudio sobre la cadena de valores en la gestión de costos en una cooperativa agrícola en el estado de Paraná en Brasil. Vesco, Tarifa, Pacheco \& Dall asta (2014), identifican los elementos que conforman la cadena de valor y su efecto en las etapas de ejecución, planificación y control de costos, mediante un análisis estratégico y competitivo, lo que permite desarrollar una ventaja sostenible por medio de controles relacionados con los competidores en el área, la cual es considerada la de mayor importancia en la toma de decisiones. Entre estos componentes analizados, se encuentran los proveedores de materias primas, la logística de entrada de productos y la logística de salida de productos con un $100 \%$ de respuestas; los servicios posventa un $81 \%$, las ventas a clientes, $56 \%$ y las operaciones con productos un $44 \%$. Jerez, Palacios \& Castro, describen la relación entre la ventaja competitiva y los resultados empresariales en un contexto de responsabilidad social, bajo un enfoque teórico basado en recursos y capacidades.

Asimismo, en el mismo país, se encuentra a do Nascimento, Calle-Collado \& Benito (2020), quienes analizan la contribución a la sostenibilidad de la agricultura familiar a partir de la Economía Social y Solidaria ESS, así como el único elemento que hace parte de la cadena de valor entre el agricultor y el consumidor, la comercialización, que afecta la sostenibilidad económica, por la cual obliga al uso de canales cortos los cuales no producen iguales beneficios, no obstante, es la única opción en el marco de una cadena de producción sostenible.

Para el caso de los pequeños productores de café, requieren la implementación de estrategias en el campo de producción y comercialización para competir en el mercado, es así como la India Coffee Board ha tomado la decisión de apoyar a estos pequeños productores en el campo y en los procesos de exportación, además apoyar a través de estrategias de mercado y tecnología que permite que ingresen en la cadena de valor mundial, entre las que se encuentra la cadena de bloques para venta on line, además facilidad en el acceso al comercio electrónico permitiendo la conexión con las plataformas más importantes en la India en las cuales pueden llevar a cabo directamente el proceso de comercialización. Otra estrategia implementada, se encuentra en el apoyo en la 
producción de cafés eco-certificados que les otorga mayor rendimiento por su costo en el mercado.

\section{DISCUSIÓN}

La literatura consultada permite concluir que si bien la expresión "desarrollo sostenible", se formalizó desde 1987, un poco más de treinta años no se ha logrado su implementación en diversos campos. La inclusión de la responsabilidad social empresarial RSE da otro vínculo que resalta la importancia de que los procesos se dirijan hacia el camino de la sostenibilidad. Asimismo, todo lo concerniente a la normatividad y los principios en el marco de la Convención Marco sobre Ética del Turismo, deben permitir una dinámica muy diferente a lo que se viene trabajando, originándose la pregunta de qué tanto se aplican dichas normas, ¿hasta dónde desconocer las normas se convierte en excusa para no progresar?

De nuevo en el turismo sostenible, aunque se identifican la falta de incentivos, también se encuentran afirmaciones que identifican a la sostenibilidad como elemento clave para todo tipo de desarrollos; ya en los estudios de casos en contextos diferentes, se puede determinar un común denominador, la nula, escasa o ausente participación gubernamental que permitiría incrementar los efectos positivos en estas zonas donde la misma población y otras entidades ya han iniciado diversas labores y se encuentran ya en el camino del desarrollo sostenible apropiándose de lo que esto implica. Por el tema de la cadena de valor, los estudios comprueban la importancia de su uso, en cualquier etapa del proceso; la selección de los factores a medir depende de la dinámica de la empresa, así como de lo que desea optimizar el líder de la misma. En este caso, la pregunta es, por qué si se han establecido cadenas de valor en diversos campos del sector agrícola y se evidencia sus aportes, ¿por qué no se han planteado los investigadores realizar análisis puntuales que determinen una cadena de valor agrícola local?, como se dijo anteriormente para este caso en particular, no se encuentran estudios para la ciudad de Bogotá.

Finalmente, describir la relación entre turismo y agricultura permite concluir que un buen direccionamiento hacia el desarrollo sostenible, lograría el impulso de muchos campos, así como mejorar los estilos de vida en un país que su gran mayoría depende de la agricultura; por esto, surge como último interrogante, $i$ siendo considerada en ciertos períodos como uno de los mejores destinos turísticos la ciudad de Bogotá, por qué no se ha dado importancia a los efectos que tienen las actividades turísticas sostenibles en la cadena de valor agrícola local?

\section{REFERENCIAS}

Aboah, J., Wilson, M., Rich, K, \& Lyne, M. (2019). Operationalising resilience in tropical agricultural value chains. Supply Chain Management, 24(2), 271-300. Retrieved from https://ezproxy.unicolmayor.edu.co:2136/doc view/2190428563/fulltextPDF/A06AF217666 94546PQ/1 ?accountid $=50438$

Addinsall, C., Weiler, B., Scherrer, P. \& Glencross, K (2017). Agroecological tourism: bridging conservation, food security and tourism goals to enhance smallholders' livelihoods on South Pentecost, Vanuatu, Journal of Sustainable Tourism, 25(8), 1100-

1116, DOI: $10.1080 / 09669582.2016 .1254221$

$$
\text { Retrieved }
$$

https://www.tandfonline.com/doi/full/10.1080 /09669582.2016.1254221;

https://epubs.scu.edu.au/cgi/viewcontent.cgi? article $=1800 \&$ context=bus_tourism_pubs

Alcaraz, José \& Susaeta, Lourdes \& Suarez, Esperanza \& Colon, Carlos \& GutiérrezMartínez, Isis \& Cunha, Rita \& Leguizamón, Francisco \& Idrovo, Sandra \& Weisz, Natalia \& Faia Correia, Manuela \& Pin, José. (2017). The human resources management contribution to social responsibility and environmental sustainability: explorations from Ibero-America. The International Journal of Human Resource Management. 124. 10.1080/09585192.2017.1350732. Retrieved from https://www.researchgate.net/publication/318 669406_The_human_resources_management _contribution_to_social_responsibility_and_e nvironmental_sustainability_explorations_fro m_Ibero-America

Amaya, I., Guzmán, F., Salazar, L. Turismo sostenible desde la responsabilidad social empresarial caso de estudio en el grupo hotelero Londoño (GHL) de Colombia. Revista TURPADE, Revista de Turismo, Patrimonio y Desarrollo, 8. Recuperado de http://www.turpade.com/f/FG60.pdf

Ambelu, G., Lovelock, B. \& Tucker H. (2018). Empty bowls: conceptualising the role of tourism in contributing to sustainable rural food security, Journal of Sustainable Tourism, 2(10), 1749-

1765, DOI: $10.1080 / 09669582.2018 .1511719$ 
Retrieved

from

https://www.tandfonline.com/doi/abs/10.1080 /09669582.2018.1511719

Andrade, S. G. (2015). Cadena de valor económico del vino de Baja California, México. Estudios Fronterizos, 16(32), 163-193. Retrieved from http://www.scielo.org.mx/scielo.php?script=s ci_arttext\&pid=S0187-69612015000200006

Blanco Rangel, I. (2016). Apuntes sobre Colombia a propósito de su internacionalización y otros desafíos. Aglala, 7(1), 209-224. Recuperado de

http://revistas.curnvirtual.edu.co/index.php/ag lala/article/view/905

Babu, P., Gokavi, N., Rudragouda, C. \& Reddy, Y. (2019). Value chain upgrading strategies for integration of indian small coffee growers in global coffee value chain. Economic Affairs, 64(4), 717-723. Retrieved from https://ezproxy.unicolmayor.edu.co:2136/doc view/2346713993/fulltextPDF/424A7496946 449CAPQ $/ 1$ ? accountid $=50438$

De Bon, H., Parrot, L., Moustier, P. (2010). Sustainable urban agriculture in developing countries. A review, Agron. Sustain. Dev., DOI: 10.1051/agro:2008062. Retrieved from https://www.agronomyjournal.org/articles/agro/abs/2010/01/a7160/a 7160.html

Bernal Payares, O. (2018). Planeación estratégica y sostenibilidad corporativa. Conocimiento Global, 3(1), 50-55. Recuperado a partir de http://conocimientoglobal.org/revista/index.p hp/cglobal/article/view/27

Castro Alfaro, A. F. (2018). Bienestar como fuente de actuación organizacional. Enfoque Disciplinario, 3(1), 1-18. Recuperado a partir de

http://enfoquedisciplinario.org/revista/index.p hp/enfoque/article/view/10

Cardona Arbelaez, D., Lamadrid Villarrreal, J., \& Brito Carrillo, C. (2018). La gestión y dirección del talento humano desde el análisis sobre clima organizacional y sus dimensiones. Un estudio de caso. Aglala, 9(1), 154-176. Recuperado de http://revistas.curnvirtual.edu.co/index.php/ag lala/article/view/1185

Castellanos J, Alvarado R.T., Aranguren Zambrano S. (2015). Diseño de Estrategia de Control Avanzado para Sistema de Celdas de Flotación en el Tratamiento de aguas de Producción de Petróleo y Gas. Revista Tecnologías de Avanzada, ISSN: 1692-7257

García Guiliany, J., Paz Marcano, A., \& Cantillo Campo, N. (2019). Estrategia y habilidades para la competitividad: caso de pymes del sector construcción en Barranquilla. Aglala, 10(1), 312-339. Disponible en http://revistas.curnvirtual.edu.co/index.php/ag lala/article/view/1349

do Nascimento, F., Calle-Collado, Á., \& Benito, R. (2020). Economía social y solidaria y agroecología en cooperativas de agricultura familiar en brasil como forma de desarrollo de una agricultura sostenible. CIRIEC Espana, (98), 189-211,189A. Recuperado de http://ciriec-revistaeconomia.es/wpcontent/uploads/CIRIEC_9807_Schwab_et_al .pdf

Gómez Bustamante, E., Bohórquez Moreno, C., \& Severiche Sierra, C. (2018). Socialdemographic and health conditions in waste pickers in the city of Cartagena de Indias (Colombia). Aglala, 9(1), 430-442. Recuperado de http://revistas.curnvirtual.edu.co/index.php/ag lala/article/view/1237

González, C., Palacios, J., \& Perea J. (2017). Business intelligence and its role in organizational agility. Criterio Libre, 15(26), 239-258. https://doi.org/10.18041/19000642/criteriolibre.2017v15n26.1049

Guzmán, F., Amaya, I., Salazar, L., Ramírez, M. (2018). Responsabilidad social empresarial en turismo, un caso práctico en la cadena hotelera GHL. En La Responsabilidad Social: académica, corporativa e innovadora (p.20). Bogotá: Universidad ECCI. Recuperado de https://www.ecci.edu.co/sites/default/files/20 19-03/responsabilidad-social-academicacorporativa-innovadora.pdf

Instituto Distrital de Turismo IDT (2019), Identificación Producto Bogotá. Recuperado de

http://www.bogotaturismo.gov.co/sites/defaul t/files/PRODUCTO BOGOTA 2018-2019.pdf

Jerez, G., Palacios, J., Castro, A (2018). Responsabilidad Social Corporativa generadora de resultados Empresariales y fuente de ventaja competitiva: capital intelectual. En Perea, J., Palacios, J., Olmedo, E. La Responsabilidad Social: Académica Corporativa e innovadora (pp. 15-42). Bogotá D.C, Colombia, Editorial Scientometrics E Researching Consulting Group

Lalangui, J., Espinoza Carrión, C. R., \& Pérez Espinoza, M. J. (2017). Turismo sostenible, un aporte a la responsabilidad social empresarial: Sus inicios, características y desarrollo. Universidad y Sociedad [seriada 
en línea], 9(1), pp. 148-153. Recuperado de https://rus.ucf.edu.cu/index.php/rus/article/vie w/498/pdf

Molina-Azorín, J. F., \& Font, X. (2016). Mixed methods in sustainable tourism research: An analysis of prevalence, designs and application in JOST (2005-2014). Journal of Sustainable Tourism, 24(4), 549-573. doi:10.1080/09669582.2015.1073739.

Retrieved from https://www.tandfonline.com/doi/full/10.1080 /09669582.2015.1073739

Niño, S, \& Contreras, M (2020). Actividad turística en el parque natural chicaque como factor de desarrollo sostenible en las veredas chicaque y cascajal (cundinamarca, colombia) // tourist activity at the chicaque natural park as a factor of sustainable development in the chicaque and cascajal zones (cundinamarca, colombia). Turismo y Sociedad, 26, 173-203. Recuperado

de https://ezproxy.unicolmayor.edu.co:2136/doc view/2319668660?pq-origsite=summon

Oyejide, T. A., Ogunkola, E. O., \& Bankole, A. S. (2019). Building and integration of agricultural value chains in Nnigeria. Canadian Foreign Policy, 25(3), 254-268. Retrieved from https://www.tandfonline.com/doi/abs/10.1080 /11926422.2019.1590216

Pachón, F., Bokelmann, W. \& Ramírez, C. (2017). Heritage and patrimony of the peasantry framework to address rural development and its application in Colombia. Acta Agronómica, 66(3), 347-359. https://dx.doi.org/10.15446/acag.v66n3.60949 . Retrieved from http://www.scielo.org.co/scielo.php?script=sc i_arttext\&pid=S0120-28122017000300347

Paz-Marcano, A., Castro-Alfaro, A., \& PalaciosRozo, J. (2019). Competencias del Emprendedor Social Aliado de las Comunidades del Entorno Empresarial Petrolero Venezolano. Saber, Ciencia $Y$ Libertad, 14(2), 163-177. https://doi.org/10.18041/23823240/saber.2019v14n2.5883

https://revistas.unilibre.edu.co/index.php/sabe r/article/view/5883/5462

Lagos, F., Sánchez, F. \& Palacios, J. (2019). Gestión para la sostenibilidad en el centro histórico de Mompox-Colombia. Espacios, 38(40), $8 . \quad$ Recuperado de https://www.revistaespacios.com/a19v40n38/ 19403808.html
Pulido-Fernández, J. I., \& María de la Cruz PulidoFernández. (2015). ¿Sigue vigente el paradigma del turismo sostenible? reflexiones a la luz de la literatura reciente/Is the sustainable tourism paradigm still valid? reflections in light of recent literature. Pasos, 13(6), 1315-1335.

Salom, R. P. (2020). Los convenios internacionales y la promoción internacional del turismo sostenible. Anuario Español De Derecho Internacional, 36, 105-157. Retrieved from https://ezproxy.unicolmayor.edu.co/docview/ 2398699197 ? accountid $=50438$

Scott, G. (2014). Agregando Valores a Las Cadenas De Valor. RAE: Revista de Administração de Empresas, 54(1), 67-79. Retrieved from https://www.scielo.br/scielo.php?script=sci_a rttext\&pid=S003475902014000100007\&lng=es\&tlng=es

Serrano-Amado, A., Montoya-Restrepo, L., \& Cazares, I. (2018). Analysis of the sustainability and competitiveness of tourism in colombia. Gestión y Ambiente, 21(1), 99109. Retrieved from https://ezproxy.unicolmayor.edu.co:2136/doc view/2091214127?pq-origsite $=$ summon

Veldkamp, A., Altvorst, A., Eweg, R., Jacobsen, E., Kleef, A., Latesteijn, H., et al. (2009). Triggering transitions towards sustainable development of the Dutch agricultural sector: TransForum's approach. Agronomy for Sustainable Development, 29(1), 87-96. doi:10.1051/agro:2008022. Retrieved from https://www.agronomy-

journal.org/articles/agro/abs/2009/01/a8002/a 8002.html

Vesco, D., Tarifa, M., Pacheco, V. \& Dall asta, D. (2014). Cadeia de valores na gestão de custos: uma análise estratégica em cooperativas agropecuárias paranaenses. Revista IberoAmericana de Estratégia, 13(2), 83-98. Retrieved from http://www.spell.org.br/documentos/ver/3228 6/cadeia-de-valores-na-gestao-de-custos-uma-analise-estrategica-em-cooperativasagropecuarias-paranaenses

Weaver, D. \& Tang, C. \& Zhao, Y. (2020). "Facilitating sustainable tourism by endogenization: China as exemplar," Annals of Tourism Research, Elsevier, vol. 81(C). Retrieved from https://www.sciencedirect.com/science/article /abs/pii/S0160738320300347 